\title{
Looking to the Future
}

\author{
Peter Lachman
}

\section{Learning Objectives}

- Understand the future challenges for patient safety

- Describe how psychological safety is essential for safety

- List the social determinants of patient safety

- Comprehend the concepts of co-production of safety

- List facilitators and risks of new technologies for safety

\subsection{Introduction}

In this chapter, I will explore the issues that we need to address as we proceed on the safety journey. This will include reflections on the beliefs that have resulted in the healthcare system we have created. It is important to consider the real issues of design and whether we need to change all aspects of healthcare delivery if we really want to be safe.

It is clear that the rapid progress in medical science over the past century has resulted in untold benefits for all. Foucault [1] described the emergence as the development of the "clinical gaze", whereby the person became a patient with a disease, so was no longer a person, but rather a

P. Lachman $(\bowtie)$

International Society for Quality in Health Care (ISQua), Dublin, Ireland "clinical diagnosis" subject to tests and interventions. As a result, the needs of the person were changed, and their narrative was not as important as the medical tests and investigations. There have been many benefits in the development of modern medicine and the science that was created to provide successful interventions. People who became patients were cured or provided longevity. This in turn has resulted in the new challenges of chronic disease and the ageing population [2]. Unfortunately, in many societies, there are both the old problems of infection and late treatment of disease, as well as the new problems of ageing and chronicity. In addition, economic and political decisions have created a vast challenge of poverty-related healthcare with poorer outcomes.

Alongside the technological advance, we also have the loss of the compassionate part of healing which has had a major impact on the psychological safety of healthcare providers and the people who receive care. The improvement in outcomes in terms of disease management has been accompanied by increasing levels of adverse events and harm. The development of the patient safety movement over the past 20 years is a reflection of the advances in healthcare and the realisation that with success came a new problem of inadvertent harm. On reflection, healthcare delivery was not planned to be safe.

As we look to the future, the healthcare industry is at a critical juncture. The rapid develop- 
ment of theories on how to deliver safe, person-centred care means that we can no longer rely on the excuse that "healthcare is different" from other industries, so cannot be reliable and safe. People are now demanding safety and reliability in the care they receive, and they want to be treated as people who happen to be ill, rather than as a number or a disease. Currently, it is by chance rather than by design that one receives highly reliable person-centred and safe care. Yet we continue to build the same type of hospitals, educate future nurses and clinicians as we have always done, and operate in a hierarchical system that disempowers people, rather than enables people to be healthy.

An examination of the patient safety movement provides an understanding of where we need to go as we plan for the future. With some imagination, we can redesign the processes of care to be compassionate and safe. Bates and Singh [3] note that there has been much progress since the publication of To Err is Human [4]. We have learnt many methods of quality improvement, and patient safety as a science has numerous theories, methodologies, and tools that, if implemented, can decrease harm: "Highly effective interventions have since been developed and adopted for hospital-acquired infections and medication safety, although the impact of these interventions varies because of their inconsistent implementation and practice. Progress in addressing other hospital-acquired adverse events has been variable" [3].

Amalberti and Vincent [5] have taken the view that the healthcare delivery system has inherent risk and that the focus of patient safety should be on the proactive management of that risk. This is true of any complex adaptive system, which makes it difficult to be safe all the time [6]. However, the health system has not been designed with safety as the core function. Given that we know that there is more complexity, perhaps a total redesign of the system is the way we need to go as we move to the future.

Although the provision of healthcare is complex, it is possible to overcome the complexity and provide care that is of the highest standard in all the domains of quality. To achieve a safe system, we will need to address some fundamental issues that we have accepted as the norm.

\subsection{The Vision for the Future}

The future vision is often reflected in the concept of Zero Harm. There are movements to apply the standards to medicine that we accept in other industries. The argument for and against zero harm is compelling. If we do not aim for zero, what is the number we need to aim for? It may be that we aim for zero in some specific areas while accepting that within the complexity of the healthcare zero, the totality of zero is a mirage, one that we need to aim for but will never reach. It has been argued that the ideal of Zero Harm is unrealistic [7], that we should accept the inherent risk in the delivery of healthcare and therefore actively adopt patient safety initiatives to improve outcomes and minimise risk. Furthermore, we need to accept the stresses healthcare systems face in the delivery of care-be it of demand, finance, or morale.

\subsection{The Challenges to Overcome to Facilitate Safety}

The pursuit of a healthcare system that is safe will require courage, as the current power base is not conducive to safe care. The power of the medical profession, pharmaceutical industry, and supporting bodies is based on the current model of care, with hierarchies and structures. Hospitals, as a concept, gained their power in the last century and were developed for the illnesses that we have now addressed, so the next stage is to integrate that power with the wider health community. This will result in changing the power imbalance in the system and the recognition that the design of a system with the hospital at the centre can be changed to the hospital as the facilitator of health within a system of care delivery which is focused closer to the home. This will require a reallocation of resources to primary care and a change of healthcare to health. There 
is a way forward to address these key issues and there is hope that in time healthcare delivery and the promotion of health will be safe with proactive minimisation of risk. People will still be harmed; however, the degree of harm will be different to the current situation. As we redesign services to be safer in the future, we will need a vision that sees beyond the current challenge and plans for an integrated service of care focused on health rather than disease (Box 4.1).

\section{Box 4.1: Changes for the Future}

1. Develop the language and culture of safety

- Use the right language about safety

- Leaders ask the right questions about safety

- Educate people for safety

\section{Promote psychological safety}

- Care for both physical and psychological safety of people

- Nurture providers of care and provide meaning in work

- Ensure that providers of care have a sense of belonging

- Listen to and hear person stories

\section{Design for safety}

- Invest in health rather than healthcare

- Co-produce safety with people not with patients

- Place people in charge of their health, not their disease

- Use human factors to address complexity

4. Social determinants for Patient Safety

- Recognise the importance of social determinants of health and their impact on safety

- Care is culturally sensitive and promotes safety

5. Harnessing technology for the future

- Digital health for safety

- Empowering people with technology

\subsection{Develop the Language and Culture of Safety}

- Use of the language that enhances safety

- Leaders asking the right questions about safety

- Educate people for safety

As healthcare is a complex system, so is the culture which is manifest within any organisation. Culture defines our belief systems and in turn how we behave. Within any organisation this will be complex, with differing safety cultures [8]. The culture we represent is evident in the language we use. Patient safety is the current terminology and as we move to a more people-centred approach, the language we use will evolve to being people centred rather than patient focused. Language reflects culture, so if we want to develop a safety culture, then we will need to critically analyse the terminology we use. Healthcare is a misnomer as it focuses on disease management, whereas we need to focus on health and the maintenance of both physical and mental well-being. Patients will be protected if we view them as people with a disease, with a life outside the disease, rather than as patients with a disease. This results in a loss of power and control over their own lives and lack of power may be a contributing cause of harm.

Patient Safety is the overall science, Risk Management was the first intervention that was developed in the safety journey. In essence, this was not about managing risk but rather about managing incidents that had occurred. While this is essential, it has not resulted in a decrease in harm and the learning from it has not been as great as it should be. The move to learning from investigation has been one of the greatest challenges we have faced. If one considers the integration of resilience engineering into the risk management approach, then the incident investigation will be a study of work as it really is over the pathway and not the incident. We now need to move to the concept of looking at the patient journey and how health is provided, so that the person is protected at all parts of the journey [9]. Management of risk is a proactive activity and is what should happen at 
all times, not only when there is an incident. It implies the acceptance of risk rather than the desire to eliminate risk, and constant mitigation will decrease the potential for harm.

Another example of language ambiguity is the term, "near misses", which is used for when we nearly harm a person but then either due to the action of an individual, or by chance the person is not harmed. This is really a near hit and if it were termed as such, perhaps we would pay more attention to the problem.

Leadership for safety will be the foundation of future work in patient safety. Leaders in healthcare are at all levels in the system, as there needs to be a focus in every microsystem as well in the de facto leadership at executive level. This includes the appreciation of uncertainty, the integration of information from different sources and the setting of the goals that will allow for the development of safe systems. Leadership, therefore, needs to be encouraged at all levels of the organisation, with the development and facilitation of local leadership at the interface with the patient as the key to ensure that there will be a safe environment. Change will require leaders who understand what quality, safe person-centred care really is, with a deep understanding of Systems Theory and Human Factors, as well as knowing how to realign the budget to facilitate change. This requires vision to set the direction, hope to provide succour in trying times, respect for what is being changed and for the work that is done, and courage to make the changes against the resistance that the past ways will present.

At a policy level, the wider implication of a total redesign of the system will require political will to allow the realignment and re-engineering of the healthcare system to one in which all policy is aimed at the long-term health of the community. Politicians need to invest in health while funding healthcare.

All of this change will require courage and imagination, vision, and hope. But more importantly, it will require co-production with all the people involved, particularly people who will be receiving care. The patient safety movement has tended to apply tools and methods to people, rather than designing with them. This implies the need to be open and transparent with patients and their families.

The healthcare workforce will require an education that enables them to deliver health as well as manage disease safely. This will require an understanding of the theories of Complexity Science, Systems Theory, Patient Safety Science, and Human Factors. Medical curricula must be challenged and changed to educate the clinicians that we require in the future [10].

\subsection{Promote Psychological Safety}

- Care for both physical and psychological safety of people

- Nurture providers of care and provide meaning in work

- Ensure that providers of care have a sense of belonging

- Address the challenge of clinician burnout

Psychological safety is the foundation for providing safe care for individuals. The work by Edmondson has led the way to understanding that, in order to deliver safe care, we need to engender the "psychological safety" of individuals in the health workplace, so that they in turn are part of the overall culture of safety. Edmondson defines psychological safety as a "shared belief held by members of a team that the team is safe for interpersonal risk-taking" [11-13].

The safety movement has called for organisations to facilitate safety culture, in which individuals have responsibilities to be safe and to carry out their work in a manner that will mitigate against harm. Given the complexity of the type of work undertaken in healthcare, this is difficult to achieve within the current hierarchical constructs of most health organisations.

While some hierarchy is essential, the ability to take risks and feel able to challenge in order to promote safe practices is one of the major challenges we will face going forward. Investigations of clinical incidents usually identify communication issues in which hierarchy prevents the communication of potential risk, teamwork being 
problematic and blame being present. The concept of psychological safety is now central to the development of safe systems, and is therefore as important as the development of tools and methods to facilitate safe care. Much of the concepts of building resilience in healthcare organisations will require attention to how we support all members of staff to be part of teams with a sense of belonging in which the meaning of work includes safety of the individuals, supported to challenge and able to learn in real time.

The concepts of safety need to build the resilience by also learning from what works within the complexity of care delivery to address the wellbeing of clinicians [14]. Included in the development of a safe environment will be an active programme to prevent burnout of clinical staff as this has a negative impact on both their well-being and the safety of patients. Prevention of burnout has not been part of the traditional patient safety interventions, yet stressed clinicians are unable to deliver safe care. Interventions. As we take a systems and human factors approach to patient safety, part of that approach will be the management of burnout taking into account the multifactorial reasons from education, hierarchies, technology, and overall design of the service [15].

The progress made in development of interventions will now be matched by the concept that the delivery of healthcare requires the concept of patient safety is our core business and all that we do need to be focused on safety. Therefore, all people working in the healthcare setting need to be supported to be safe and to proactively work to their own safety from a psychological and physical perspective. The safety of the people for who they care will then follow.

\subsection{Design for Health and for Safety}

- Invest in health rather than healthcare

- Co-produce safety with people not with patients

- Place people in charge of their health, not their disease

- Use human factors and ergonomics to address complexity
The patient safety movement has been focused on healthcare which really implies that it is concerned with the negative impacts in the management of disease. The future of the movement will transcend disease and focus on maintaining the health of people, even when they have disease. This approach implies that people with a disease need to have their physical and mental health beyond their disease protected at all times by minimising the risk of harm. To achieve this aim, we need to move to a new paradigm, and change the current design of our healthcare system, which is focused on physiological systems rather than the person as a whole. This implies a change in the systems we have created, which have been medically focused. It does not imply that we destroy all we have, but rather that we examine people flows, human factors, and safety from the eyes of the person receiving care.

The concept of engaging with the people who receive care has become central to the personcentred care movement. The person-centred care approach is more than asking about satisfaction and experience, but rather in sharing responsibility for health and becoming partners in healthcare provision. The realisation that we cannot be safe without the involvement of the people who we care for in the planning and design of services has led to the concept of co-production, in which people are part of the solution rather than part of the problem [16-18]. This approach implies a radical rethink on how we define adverse events, how we look at harm from the viewpoint of the family and person harmed, and how we investigate safety incidents with the inclusion of the patient as a person, not as a patient. It will require a re-evaluation of clinical risk, a change in the power imbalance and real consultation with people about risk and the relative benefit of intervention. Co-production also implies that we co design safety not only with the people we call patients, but also with the providers of care who have to be safe all the time, despite the inherent risk of the clinical processes and especially in trying conditions.

To achieve safety within clinical process will require the integration of safety design as part of the day-to-day operations. Human Factors and Ergonomics (HFE) has been a marginal topic in 
healthcare, pursued by enthusiasts rather than being core to the programmes that we run. In other chapters, the HFE theories have been presented. HFE will be as integral to medical education as anatomy and physiology, so that it is a seam that runs through all of our thinking [19].

\subsection{Social Determinants of Patient Safety}

- Define the importance of social determinants of patient safety

- Design care that is culturally sensitive and promotes safety

In recent years, the importance of the determinants of health outcomes has been highlighted with the studies that indicate that people who are less well-off economically, are from ethnic minorities or marginalised groups have worse health outcomes. Poverty, housing, education, literacy, and nutrition are a few of the factors that interplay to cause clinical presentations, as well as the outcome of treatment be it due to poor access, lack of health literacy, or institutionalised prejudice [20]. Health outcomes can be predicted depending on the influences of the social determinants. The poorer one is, the worse is the clinical outcome. Poverty influences life expectancy, the type of diseases to which one will succumb, the access to health, and the quality of healthcare [21]. The patient safety movement has not traditionally researched the impact of the social determinants of health on the risk of harm, either for individuals or for communities [22].

It is logical to expect that people who are poor, have low health literacy, and do not have equal access to the healthcare system, are likely to be at risk of harm due to the immense power differentials and the institutionalised prejudice they experience. If one adds ethnicity, gender, language and status, e.g. refugee or homeless, then the outcome is likely to be even worse. The challenge for the patient safety movement is to acknowledge the inequity and to mitigate against it in the design of programmes. We need to measure this perspective of patient safety in order to allow for the development of interventions that empower people and address the impact poverty and disadvantage have on safety.

From a global perspective, the work by the Lancet Commission on the increased risk to the people in the poorer nations of the Lower and Middle Income Countries indicates that we will need more than the patient safety methodologies to protect people in those countries from harm [23]. In the future, the Social Determinants of Patient Safety (or SDPS) will be as important in understanding how to prevent harm as are the methods and interventions we use to mitigate against adverse events.

\subsection{Harnessing Technology for the Future (Reference Chap. 33)}

- Digital health for safety

- Empowering people using technology

- Understand the opportunities and risks of Artificial Intelligence

The challenge we face in the patient safety movement is how we harness the great potential of the digitalisation of health and the introduction of Artificial Intelligence to healthcare delivery. The potential to use new technologies to design out human medical error and resultant harm is great. Nonetheless, it is not necessarily the solution to the challenge of patient safety, but rather an aid towards safer care [24, 25]. The potential of Electronic Health Records, electronic prescribing, and computer ordering systems to address the communication and transcription challenges in patent safety are still to be realised. They have not overcome communication issues, but offer us the opportunity to have better communication and easier pathways, if the implementation is successful. The challenge is to not replace old errors with new ones [26]. As most of the implementation has been in upper income countries, the spread of digital solutions around the globe will need to be carefully considered to ensure that the lessons learnt are applied with the safety of patients at the core. 
For people receiving care, the use of smart phone technology can empower them to manage their care with ready access to information, medical records, test results, and control of their own data. This will require careful development, with ceding of power from the professionals to the people receiving care. Co-production of safety solutions will be an essential part of realising the potential of technology.

Artificial Intelligence (AI) has the potential to fundamentally change the way we care for people and to enhance the safety of care. However, the future development and implementation will need to address numerous challenges, such as the reliability of the predictions made from the newly developed machine learning systems. The transferability of the information and how data matches complexity of different health systems and how we as clinicians interact with the new technology [27]. While it is still early in the development of AI solutions that can assist us in our safety journey, we in the patient safety field must join with AI developers to harness the potential of predictive modelling in the future. The safety movement will need to be integral to the development of AI solutions and ensure that there is a safer system in the future [28].

\subsection{Conclusion}

We have come a long way in the patient safety movement. The standards of care in the past have focused on the processes of care and now need to be redesigned by people who receive care. This will change their focus from measurement of process to a refined assessment of people's experiences and the desired outcomes. Patient safety in the future will not be about the interventions needed, but rather about the people who work in the system, the people who receive care, and how we can design systems to support them in the delivery of reliable and safe care.

If we truly want to aim for Zero Harm as a concept, then we will need to redesign our systems of care through co-production and partnership, and address the challenges of the social determinants, the hierarchical cultures, and the opportunities of technology.

\section{References}

\section{Overview}

1. Foucault M. The birth of the clinic. An archaeology of medical perception. Paris: Vintage; 1994.

2. Amalberti R, Vincent C, Nicklin W, Braithwaite J. Coping with more people with more illness. Part 1: the nature of the challenge and the implications for safety and quality. Int J Qual Health Care. 2019;31(2):154-8.

3. Bates DW, Singh H. Two decades since to err is human: an assessment of progress and emerging priorities in patient safety. Health Aff. 2018;37(11):1736-43.

4. Donaldson MS, Corrigan JM, Kohn LT. To err is human: building a safer health system. Washington DC: National Academies Press; 2000.

5. Amalberti R, Vincent C. Managing risk in hazardous conditions: improvisation is not enough. BMJ Qual Saf. 2020;29:60-3.

6. The Health Foundation. Research scan: complex adaptive systems. 2010. https://www.health.org.uk/ sites/default/files/ComplexAdaptiveSystems.pdf. Accessed 27 Dec 2019.

7. Thomas EJ. The harms of promoting 'Zero Harm'. BMJ Qual Saf. 2020;29:4-6.

\section{Develop the Language and Culture of Safety}

8. Mannion R, Davies H. Understanding organisational culture for healthcare quality improvement. BMJ. 2018;363:k4907.

9. Trbovich P, Vincent C. From incident reporting to the analysis of the patient journey. BMJ Qual Saf. 2019;28:169-71.

10. Vosper H, Hignett S, Bowie P. Twelve tips for embedding human factors and ergonomics principles in healthcare education. Med Teach. 2017;40(4):357-63.

\section{Psychological Safety and Well-Being}

11. Edmundson AC, Lei Z. Psychological safety: the history, renaissance, and future of an interpersonal construct. Annu Rev Organ Psychol Organ Behav. 2014; $1: 23-43$.

12. Edmondson AC, Higgins $M$, Singer S, Weiner W. Understanding psychological safety in health care and education organizations: a comparative perspective. Res Hum Dev. 2016;13(1):65-83.

13. Edmondson AC. The fearless organization: creating psychological safety in the workplace for learning, innovation, and growth. New York: Wiley; 2018.

14. Smaggus A. Safety-I, safety-II and burnout: how complexity science can help clinician wellness. BMJ Qual Saf. 2019;28:667-71. 
15. National Academies of Sciences, Engineering, and Medicine. Taking action against clinician burnout: a systems approach to professional well-being. Washington, DC: The National Academies Press; 2019. https://doi.org/10.17226/25521.

\section{Design for Safety}

16. Batalden M, Batalden P, Margolis P, Seid M,Armstrong G, Opipari-Arrigan L, Hartung H. Coproduction of healthcare service. BMJ Qual Saf. 2016;25:509-17.

17. Batalden P. Getting more health from healthcare: quality improvement must acknowledge patient coproduction-an essay by Paul Batalden. BMJ. 2018;362:k3617.

18. Elwyn G, Nelson E, Hager A, Price A. Coproduction: when users define quality. BMJ Qual Saf. 2019. Published Online First; https://doi.org/10.1136/ bmjqs-2019-009830.

19. Hignett S, Lang A, Pickup L, Ives C, Fray M, McKeown C, Tapley S, Woodward M, Bowie B. More holes than cheese. What prevents the delivery of effective, high quality and safe health care in England? Ergonomics. 2018;61(1):5-14.

\section{Social Determinants for Patient Safety}

20. Marmot M. Social determinants of health inequalities. Lancet. 2005;365(9464):1099-104.
21. Okoroh JS, Uribe EF, Weingart S. Racial and ethnic disparities in patient safety. J Patient Saf. 2017;13(3):153-61.

22. Boozary AS, Shojania KG. Pathology of poverty: the need for quality improvement efforts to address social determinants of health. BMJ Qual Saf. 2018;27:421-4.

23. Kruk ME, Gage AD, Arsenault C, et al. High-quality health systems in the sustainable development goals era: time for a revolution. Lancet Glob Health. 2018;6(11):e1196-e252.

\section{Digital Health and Patient Safety}

24. Agboola SO, Bates DW, Kvedar JC. Digital health and patient safety. JAMA. 2016;315(16):1697-8. https://doi.org/10.1001/jama.2016.2402.

25. Sheikh A. Realising the potential of health information technology to enhance medication safety. BMJ Qual Saf. 2020;29:7-9.

26. Sujan M, Scott P, Cresswell K. Health and patient safety: technology is not a magic wand. Health Informatics J. 2019:1-5. https://doi. org/10.1177/1460458219876183.

27. Macrae C. Governing the safety of artificial intelligence in healthcare. BMJ Qual Saf. 2019;28:495-8.

28. Challen R, Denny J, Pitt M, Gompels L, Edwards T, Tsaneva-Atanasova K. Artificial intelligence, bias and clinical safety. BMJ Qual Saf. 2019;28:231-7.

Open Access This chapter is licensed under the terms of the Creative Commons Attribution 4.0 International License (http://creativecommons.org/licenses/by/4.0/), which permits use, sharing, adaptation, distribution and reproduction in any medium or format, as long as you give appropriate credit to the original author(s) and the source, provide a link to the Creative Commons license and indicate if changes were made.

The images or other third party material in this chapter are included in the chapter's Creative Commons license, unless indicated otherwise in a credit line to the material. If material is not included in the chapter's Creative Commons license and your intended use is not permitted by statutory regulation or exceeds the permitted use, you will need to obtain permission directly from the copyright holder. 